\title{
TO STUDY COMPLIANCE OF ANTENATAL WOMEN IN RELATION TO IRON SUPPLEMENTATION IN ROUTINE ANTE-NATAL CLINIC AT A TERTIARY HEALTH CARE CENTRE
}

\author{
*Dr Godara Sushila ${ }^{1}$, Dr Hooda Ritu ${ }^{2}$, Dr Nanda Smiti ${ }^{3}$, Dr Mann Sonika ${ }^{4}$ \\ ${ }^{1}$ Senior Resident, Post Graduate Institute of Medical Sciences, Rohtak, Haryana, India \\ ${ }^{2}$ Associate Professor, Post Graduate Institute of Medical Sciences, Rohtak, Haryana, India \\ ${ }^{3}$ Senior Professor, Post Graduate Institute of Medical Sciences, Rohtak, Haryana, India \\ ${ }^{4}$ Assistant Professor, Post Graduate Institute of Medical Sciences, Rohtak, Haryana, India
}

*Corresponding author's Email:drgodarasushila@gmail.com

\begin{abstract}
Background: The purpose of this study was to determine the compliance of the pregnant women and factors related to iron tablet consumption behavior among pregnant women. Response to iron deficiency anemia is related to the dose and schedule of a iron tablets and diet. Noncompliance reduces treatment benefits and is associated with poorer prognosis.

Methods: A detailed questionnaire designed to assess the antenatal women compliance. Eight hundred and sixty women of 16-30 wks pregnancy who received iron tablets daily for 90 consecutive days in Rohtak city of Haryana state.

Results: The results showed that compliance among pregnant women, regarding iron supplementation, was observed by $80.47 \%$ women, non compliance was observed by $14.42 \%$ while over compliance was observed only by $5.11 \%$ women. Compliance was better observed in educated patients as compared to uneducated i.e. $62.14 \%$ versus $37.86 \%$. The reasons for non compliance were side effects $29.03 \%$, suffering from pregnancy complications and taking multiple drug supplements $22.58 \%$, long term taking of iron tablets $19.35 \%$, not liking pharmaceutical preparations $16.94 \%$, forgetfullness $9.68 \%$ and not liking due to poor quality of iron tablet supplied by government were $2.42 \%$. Almost all the women believed that the doctor, pharmacist and nurse explained the instructions for intake of iron tablets and diet to them and they followed those instructions.

Conclusion: This study shows that compliance is related to education of the pregnant women and proper explanation of instructions by doctor, pharmacist and nurses, help to improve the compliance but much can be done to improve existing iron supplementation programs in developing countries by ensuring that iron tablets of good quality are available at all levels of health care.
\end{abstract}

Key Words: Compliance, Pregnant Women, Iron Deficiency Anemia.

\section{INTRODUCTION}

Anemia is the commonest medical disorder in pregnancy. Its prevalence may be as high as $88 \%$ in some parts of India ${ }^{1}$.Anaemia especially if severe is directly or indirectly responsible for $40 \%$ of maternal deaths ${ }^{2}$.Iron deficiency is the most prevalent single nutritional deficiency, nearly $50 \%$ of women with iron deficiency progress to iron deficiency anemia ${ }^{3}$.However, the prevalence of iron deficiency in women has decreased over past several decades and this has been variously attributed to several factors like prophylactic iron, iron supplements, better health care and public health programs, but above all, in a large measure to iron fortification ${ }^{4}$.

WHO defines anaemia as hemoglobin concentration less than 11 gram $\%$. The gross iron requirement in pregnancy is about 1240mg elemental iron that include obligate losses (230mg), red cell mass expansion (450mg), fetal requirement $(270 \mathrm{mg})$, placental tissue $(90 \mathrm{mg})$ and loss at delivery $(200 \mathrm{mg})$. A healthy pregnant woman can obtain half of this requirement from her balanced diet especially as iron absorption increases in pregnancy and about $150 \mathrm{mg}$ is saved because of amenorrhoea that leaves a net requirement of about 500mg, which can be obtained, from presumably adequate iron stores of a healthy woman. But most of our women are already anaemic, with deficient iron store sand poor dietary habits. As a result, pregnancy worsens the anemia and causes unwanted complications. The Government of India in the National Nutritional
Anemia Control Program has recommended daily intake of $100 \mathrm{mg}$ elemental iron with 500 micrograms of folic acid in the second half of the pregnancy for at least 100 days. Oral iron therapy has poor patient compliance either due to side effects or forgetfulness. In a recent study it was seen that injectable iron in two divided doses has better compliance and good results 5 .

Relation of Iron and diet - As we knows that iron taken in the form of diet which is rich in meat, grains, green leafy vegetables etc. is important in preventing iron deficiency anemia. In a diet having 2000 calorie, it is important to note that there is only about $10 \mathrm{mg}$ of elemental iron (compared to $65 \mathrm{mg}$ in one $325 \mathrm{mg}$ iron sulfate tablet). For treatment of iron deficiency anemia, dietary iron with vitamin $\mathrm{C}$ or drinking orange juice with high iron foods which enhances the iron absorption is recommended in combination with iron therapy.

Compliance may be defined as the extent to which behavior of the subject coincides with medical or health advice. It involves patient and doctor compliance. Patient compliance with drug therapy can be defined as the extent to which the patient follows a prescribed drug regimen. It involves whether the patients follows recommendations made by the provider with respect to timing, dosage, and frequency of medication taking. It also includes whether the patient follows diet schedule, attends clinics regularly or follows other instructions given by the doctor. Doctor's 
compliance is the extent to which the behavior of the doctor fulfills their professional duty i.e to prescribe accurately, to give proper instructions to the patients and to adopt new advances when they are sufficiently proved. ${ }^{6-7}$ Compliance is measured over a period of time and reported as a percentage. Compliance is a behavioral response to clinical treatment. Compliance may be complete, partial, erratic, nil or there may be over compliance. Over compliance refers to intake of drug by the patients more than prescribed, even increasing the dose by $50 \%$. Typical compliance rates for prescribed medications are about $50 \%$ with a range of $0 \%$ to more than $100 \% .^{8}$ Compliance has little relation to socio-demographic factors such as age, race, intelligence but education of the patient seems to improve compliance, and would be expected to do so. For example, reminders as diary, mobile phone, calendar pack to take the treatment can improve compliance. In addition, patients tend to miss appointments and drop out of care when there are long waiting times at clinics or long time lapses between appointments. Finally, compliance decreases as the complexity, cost, and duration of the regimen increase. As treatment response is related to the dose and schedule of a therapy, noncompliance reduces treatment benefits and can affect assessment of the effectiveness of treatments. ${ }^{9-11}$ In addition to its potential for undermining the effectiveness of any treatment, noncompliance is associated with poorer prognosis. ${ }^{12}$ Numerous studies have demonstrated that inadequate compliance with prescribed medication regimens results in increased morbidity and mortality from a wide variety of illnesses, as well as increased health-care costs. ${ }^{13-14}$

To improve compliance, antenatal women needs to know the degree of the anaemia i.e she is suffering from i.e mild, moderate and severe type, symptoms of anaemia \& the medication prescribed for the treatment of the disease. She must understand the benefits of the iron, diet $\&$ all the instructions given by the doctor especially the frequency, duration and the time at which medication should be taken. She must be well aware of the side effects and any significant drug interactions with the food, alcohol or any other medicine. ${ }^{15}$ With increasing numbers of effective self-administered treatments, the need is apparent for better understanding and management of noncompliance. The problem of low compliance has posed a challenge to many nutrition program implementors and health practitioners. Studies have been conducted to experiment on new formulations of medicinal iron or modification of the regimen from daily to weekly dosage in order to increase complance to iron supplementation. ${ }^{16,17}$ It was commented that the "issue at hand is not whether a weekly iron dose is beneficial or not, but whether pregnant women are able to receive and take iron tablets as prescribed". ${ }^{18}$

To the best of our knowledge there is no research conducted to reveal the extent of this problem in Haryana. Therefore we believe that this research may show the magnitude of problem in Haryana so as to initiate intervention by the concerned authorities and the community as well.

\section{AIMS AND OBJECTIVES}

1. Compliance in relation to intake of iron supplementation and dietary advice.
2. Factors affecting the compliance in ante-natal women.(Parity, age, education, degree of anaemia, any intervention for anaemia in previous years, knowledge of anemia i.e cause, symptom, effect, treatment and prevention of anemia).

\section{MATERIAL AND METHODS}

\section{STUDY AREA}

The study site is Rohtak city of Haryana state in India i.e OPD, Gynecology and Obstetrics Department, PGIMS Rohtak and anganwari center nearest to PGIMS Rohtak .

\section{STUDY DESIGN AND POPULATION:}

This is a cross sectional study carried out on 1000 antenatal pregnant women (16wks-30wks) who are routinely attending the ante-natal clinic. Compliance in this study defined as the adherence of pregnant women to the practice of daily intake of iron tablets for 90 consecutive days.

\section{DATA COLLECTION AND MANAGEMENT:}

A structured and pretested questionnaire was used to collect the information. It was prepared in English and then translated to local language. The answer of each question had two rating scales: yes and no. The collected variables included: economic condition of the women, age, profession, education, obestetric history, antenatal complications due to medical or pregancy related causes, questionnaire on antenatal pregnant women compliance such as did she know the symptoms of iron deficiency(weakness, headache, irritability, difficulty in exercising, pallor complexion, rapid heartbeat), did you take the iron tablets as advised, did you follow the instructions given by the doctor, did she follow the oral iron supplementation and diet schedule, did you know the degree of anemia(mild, moderate, severe), did you take any intervention for treatment of anaemia in previous pregnancies( Inj. Iron $\mathrm{i} / \mathrm{m}$ or $\mathrm{i} / \mathrm{v}$, any blood transfusion), did you attended the antenatal clinic regularly. If the antenatal women stopped taking the medication whether it was due to any side effects (Nausea, vomiting, gastric upset, constipation, unpleasant taste), long term taking of iron tablets/not liking pharmaceutical preparations(tablet) but likes other preparations as liquid, forgetfullness, not liking due to poor quality of iron tablet supplied by government and did you stop the iron tablets because of suffering from antenatal complications and taking medicine for other diseases also ( AIDS, hypothyroidism, tuberculosis, heart disease etc.). Data was collected from the antenatal women and analysed. Results are represented in the form of percentages and tables. Questionnaire is attached as appendix-1.

\section{RESULTS:}

A total of 1000 pregnant women were screened and enrolled in the study between October 2011 - September 2012. Of the 1000 pregnant women screened, 840 pregnant women included in the study. Rest 160 women were not able to finish the study required, thus, were excluded in the data analysis. The reasons were: pregnant women lost interest to continue with the study, transfer of residence, miscarriage, ectopic pregnancy etc. 
Total 19 questionnaires were asked to the 840 pregnant women to check the antenatal compliance. Most of the pregnant women were in younger age group i.e 20-30 years $(65 \%)$, followed by middle and elderly age group i.e $30-43$ years $(35 \%)$. Middle and elderly women were slightly more compliant than younger age group i.e $54.62 \%$ versus $45.38 \%$. About half of the pregnant women (54\%) were homemaker. Sixteen percent were private staff while government staffs were $30 \%$. Women in private and government staffs were slightly more compliant than homemaker $55.78 \%$ versus $44.22 \%$. Out of total pregnant women $60 \%$ were educated and $40 \%$ uneducated. The compliance were better observed in educated women as compared to uneducated i.e 62.14 vs $37.86 \%$. Multiparous women were more compliant than nulliparous i.e $59.39 \%$ vs $40.61 \%$. According to socioeconomic status $30 \%$ pregnant women belonged to middle socioeconomic group, $41 \%$ from low socioeconomic group whereas only $29 \%$ were from higher socioeconomic strata. Compliance was best observed in high socioeconomic group of women as compared to middle and lower socioeconomic group i.e. $45.38 \%$ versus $29.77 \%$ versus $24.85 \%$ respectively as shown in table 1.

Pregnant women with pregnancy complications due to antenatal causes and medical causes were slightly less compliant than routinely antinatal care pregnant women $78.5 \%$ vs $80 \%$. The pregnant women attended the antenatal clinic regularly were $76 \%$. Most of the pregnant women $(80 \%)$ understood the symptoms of anemia they were suffering from, only $18 \%$ had knowledge about the degree of anemia i.e mild, moderate and severe. The women having the knowledge about anemia were $27 \%$. Almost all pregnant women believed that doctor had nicely explained about the iron deficiency anemia, the anticipated benefits from the iron tablets and the instructions for the intake of iron tablets and diet. $80 \%$ of the pregnant women believed that the pharmacist and nurse explained the instructions for intake of medicine and they followed also.

Those pregnant women who followed the instructions, took the iron tablets and diet as advised were $80.47 \%$ (compliance). The pregnant women who stopped taking the medication because of side effects were $29.03 \%$ (Gastric upset 10.2\%, vomiting $4.6 \%$, nausea $8 \%$, constipation $4.4 \%$, unpleasant taste $2.1 \%$ ) and due to suffering from pregnancy complications and taking multiple drug supplements were $22.58 \%$,long term taking of iron tablets were $19.35 \%$, not liking pharmaceutical preparations were $16.94 \%$, forgetfullness were $9.68 \%$ and not liking due to poor quality of iron tablet supplied by government were $2.42 \%$ respectively (non compliance) while $5.11 \%$ of the pregnant women increased the dose of iron tablets themselves for additional benefits or to have quicker response (over compliance) as shown in table 2 and 3.

Table 1: Compliance of pregnant Women according to Sociodemographic profile:

\begin{tabular}{lcc}
\hline Characterstics & Number & Percentage(\%) \\
\hline Age in years & 314 & 45.38 \\
$\quad 20-30$ & 378 & 54.62 \\
$\quad 30-40$ & & \\
Occupation & 306 & 44.22 \\
Homemaker & 386 & 55.78 \\
Working private/govt. & & \\
Education status & 430 & 62.14 \\
Educated & 262 & 37.86 \\
Uneducated & & \\
Parity & 281 & 40.61 \\
Nulliparous & 411 & 59.39 \\
Multiparous & & \\
Socioeconomic status & 314 & 45.38 \\
High & 206 & 29.77 \\
Middle & 172 & 24.85 \\
Low & &
\end{tabular}

TABLE 2 Compliance in relation to oral iron and diet

\begin{tabular}{lcc}
\hline Antenatal Women & Number & Percentage (\%) \\
\hline Compliant & 692 & 80.47 \\
Non Compliant & 124 & 14.42 \\
Over Compliant & 44 & 5.11
\end{tabular}

TABLE 3 Frequency and percentage of reasons of non compliance of pregnant women regarding iron supplementation

\begin{tabular}{lcc}
\hline Causes & Frequency & Percentage (\%) \\
Side effects & 36 & 29.03 \\
Suffering from pregnancy complications & 28 & 22.58 \\
Long term taking of iron tablets & 24 & 19.35 \\
Not liking pharmaceutical preparations & 21 & 16.94 \\
Forgetfulness & 12 & 9.68 \\
Poor quality of tablet supplied by govt. & 3 & 2.42
\end{tabular}


Compliance with iron supplementation plays a major role in the prevention and treatment of iron deficiency anemia particularly among pregnant women whose iron requirement starts at the second trimester and progresses until the third trimester. Eight hundred forty pregnant women in the City of Haryana participated in the present study to determine compliance with iron supplementation as well as factors that may have influenced compliance. Pregnant women compliance describes the degree to which a women correctly follows medical advice. Noncompliance is a major obstacle to the effective delivery of health care. Prevalence of compliance in this study was $80.47 \%$ as these pregnant women understood and strictly followed the instructions given by the doctor while $5.11 \%$ of the pregnant women were over compliant and $14.42 \%$ of the pregnant women were non-compliant but the study conducted by Lertmulligaporn $\mathrm{N}$ found that the compliance rate was about $75 \%{ }^{19}$

In the present study, elderly and middle women were slightly more compliant than younger age group. The reason for this is that they may be more concerned about their health than younger patients and they get necessary support and cooperation from their family members and had better behaviors in prevention and treatment of iron deficiency anemia in pregnant women. . The findings of our studies were in accordance with study shown by Boonserm K. ${ }^{20}$

In the present study compliance was better observed in educated and high economic status pregnant women as compared to uneducated. It may be because of the fact that women with higher educational level have better knowledge about the iron deficiency anemia and therapy and therefore are more compliant. Similar results were reported by Brand et al (1977). ${ }^{21}$ The pregnant women with good knowledge had good compliance. In our study it was observed that the number of pregnant women who stopped taking the medication because of side effects were 36 out of total 840 participated in the study $(4.28 \%)$ but in Burma only $3 \%$ of women stated that side effects were the reason they stopped taking iron supplements while $30 \%$ of women in Thailand complained of side effects while taking iron tablets. ${ }^{22}$ Although there is little evidence to suggest side effects are a major deterrent to compliance with iron supplementation, they should not be ignored and consideration of them should be part of the treatment plan. ${ }^{23}$ In our study it was observed that some women not liking due to poor quality of iron tablet supplied by government, the study done by Rao also demonstrated similar results. $^{24}$ Our study shows that $80 \%$ of the women believed that the pharmacist and nurse explained the instructions for intake of medicine to them and they followed those instructions. The study done by Sipkoff also demonstrated that the pharmacists were responsible for adjusting drug regimens as well as identifying and preventing drug-related problems. The study found that this type of pharmacist intervention improved compliance. $^{25}$

\section{CONCLUSION}

This study shows that compliance is related to education of the pregnant women and proper explanation of instructions by doctor, pharmacist and nurses, help to improve the compliance but much can be done to improve existing iron supplementation programs in developing countries by ensuring that iron tablets of good quality are available at all levels of health care. To further increase compliance of pregnant women with iron supplementation, health workers should establish a good health providerpatient relationship that can help in motivating pregnant women to regularly take their iron supplements. Complete instructions, such as the dosage and manner of taking the iron tablets, the benefits of iron tablets and diet rich in iron, the side effects that may be experienced from taking the iron tablets, and what women should do when experiencing side effects, should always be communicated to pregnant women.

ACKNOWLEDGMENT: All authors contributed in the collection and interpretation of data. There are no competing interests to declare.

\section{APPENDIX - 1}

\section{QUESTIONNAIRE}

Antinatal women compliance report

Name:

Age:

$\mathrm{W} / 0$ :

Profession:

Apparent income group: low/middle/high

Address:

Education level:

Obestetric history: Date of last menstrual period, period of gestation, parity, live issues, abortions, previous LSCS, ectopic pregancy, infertility.

Antenatal complications:

1. Medical cause: Thyroid dysfunction, anaemia, viral hepatitis, diabetes mellitis, heart diseases, STS/AIDS, tuberculosis, syphilis, malaria, asthema, haematuria etc.

2. Pregancy related: pre-eclampsia, gestational diabetes mellitis, intrauterine growth retardation, oligohydramnios, antipartum haemorrhage etc. 
1. Did you visit the antenatal clinic regularly as advised?

2. Did you know the symptom of iron deficiency anaemia ?

3. Did you know the degree of anaemia( mild,moderate,severe)?

4. Did you investigate for iron deficiency anaemia $(\mathrm{Hb})$ ?

5. Did you take any intervention for treatment of anaemia as inj.Iron or blood transfusion in previous pregancy? Yes/No

6. Did you have knowledge of anemia?

7. Do you understand how much time required for treatment of the Iron deficiency anaemia?

8. Did the doctor explain that what benefits you can have from iron tablet? Yes/No

9. Did the doctor give you some instructions for the intake of iron tablet? Yes/No

10. Do you understand and follow the instructions given by the doctors (dose, duration, schedule e.g OD, BD, HS)?

11. Did you take iron tablets and diet as advised?

12. Did you stop the iron tablet because of any side effects?

$\mathrm{Yes} / \mathrm{No}$

Yes/No

Yes/No

13. Did you stop the iron tablet due to suffering from antenatal complication? Yes/No

14. Did you stop the iron tablet because of long term taking of iron tablets ? Yes/No

15. Did you stop the iron tablet because not liking pharmaceutical preparations?

16. Did you stop the iron tablet forgetfullness?

Yes/No

17. Did you stop the iron tablet because not liking due to poor quality of iron tablet supplied by government? Yes/No

18. Did you increase the dose of iron tablet yourself for additional benefits or to have quicker response? Yes/No

19.Did the pharmacist or nurse explain the instructions for intake of iron tablets?
Yes/No

Yes/No

\section{REFERENCES}

1. ICMR Evaluation of National Nutritional Anaemia Prophalaxis Programme. An ICMR Task Study. ICMR New Delhi 1989.

2. Bhatt RV. Maternal Mortality in India. FOGSI, WHO study? J Obstet Gynec of Ind 1997; 47:201-14.

3. Viferi FE. A new concept in the controll of iron deficiency. Biomed Environ Sci1998:11:46-60.

4. Scholl TO, Hediger ML. Anaemia and iron deficiency anaemia: compilation of data on pregnancy outcome. Am J Clin Nutr1994; 59:482-501.

5. Bhatt RV. Poor iron compliance-the way out. J Obstet Gynec of Ind 1997; 47:185-90.

6. Haynes RB. Improving patient adherence: state of the art, with a special focus on medication taking for cardiovascular disorders. In: Compliance in Health Care an Research. Burke LE, Ockene IS, eds. New York, NY: Futura Publishing Co Inc; 2001:3-21.

7. Haynes RB, McDonald H, Garg AX. Interventions for helping patients to follow prescriptions for medications [Cochrane Review on CD-ROM]. Oxford, England: Cochrane Library, Update Software; 2002; issue 2.

8. Sackett DL, Snow JC. Compliance in Health Care. Baltimore, Md: Johns Hopkins University Press; 1979:11-22.

9. Gordis L. Conceptual and methodological problems in measuring compliance. In: Haynes RB, Taylor DW, Sackett DL, eds. Compliance in Health Care. Baltimore, Md: Johns Hopkins University Press; 1979:23-5.

10. Haynes RB. Determinants of compliance: the disease and the mechanisms of treatment. Haynes RB, Taylor DW, Sackett DL, eds. Compliance in Health Care. Baltimore, Md: Johns Hopkins University Press; 1979:49-62.

11. Haynes RB, Dantes R. Patient compliance and the conduct and interpretation of therapeutic trials. Control Clin Trial. 1987; 8:129.

12. Irvine $\mathbf{J}$, Baker $\mathrm{B}$, Smith $\mathrm{J}$, et al. Poor adherence to placebo or amiodarone therapy predicts mortality: results from the CAMIAT study. Psychosom Med. 1999; 61:566-575.

13. Zyczynski TM, Coyne KS. Hypertension and current issues in compliance and patient outcomes. CurrHypertens Rep 2000; 2:510-14.
14. Howell N, Trotter R, Mottram DR, Rowe PH. Compliance with statins in primary care. Pharm J. 2004; 272:1-40.

15. Topics in drug therapy in Peter. N. Bennett, Murris. J Brown editors, Clinical Pharmacology $10^{\text {th }}$ ed. Churchill Livingstone Elsevier Philadelphia. P. 5-32.

16. Cook, J., Carriaga, M., Kahn, S., Schalch, W., Skikne, B. Gastric Delivery System for Iron Supplementation. The Lancet. 1990, 335: 1136-1139.

17. Ridwan, E., Schultink, W., Dillon, D., Gross, R. Effects of weekly iron supplementation on pregnant Indonesian women are similar to those of dailysupplementation. AJCN. 1996, 63: 884890.

18. Yip, R. Iron supplementation during pregnancy: is it effective? AJCN.1996, 63: 853-855.

19. Lertmulligaporn N. Effect of daily versus weekly iron supplementation in rural northeastern pregnant women, Thailand [M.S. Thesis in Nutrition Bangkok: Faculty of Graduate Studies, Mahidol University; 2000.

20. Boonserm K. Factors affecting compliance with the prevention and treatment measure of iron deficiency anemia in pregnant women: a case study Chainat province [M.P.H.M Thesis in Family Health]. Bangkok: Faculty of Graduate Studies, Mahidol University; 1996.

21. Brand, et al. Effect of Economic Barriers to Medical Care on Patient's Non-Compliance. Public Health Reports. 1977, Vol. 92 (2), 72-78).

22. Charoenlarp P.. Dhanamitta S., Kaewvichit R.. Silprasert A., Suwanaradd C. et al. A WHO collaborative study on iron supplementation in Burma and Thailand. Am. J. clin. Nurr. 47, 280-297. 1988.

23. NCP-Nutrition Center of the Philippines-Bulletin. Jan-Mar, 1989.

24. Rao B. S. Narasinga. Prevention and control of anaemia in India. Theory and Practice. NFI Bull. 12, 4-8. 1991.

25. Sipkoff $M$. The newsletter of quality issues in health care. QualityIndicator.com June, 2001. 